\title{
Immunotherapy-Associated Cardiotoxicity of Immune Checkpoint Inhibitors and Chimeric Antigen Receptor T Cell Therapy: Diagnostic and Management Challenges and Strategies
}

\author{
Ashley F. Stein-Merlob ${ }^{1}$ - Michael V. Rothberg ${ }^{2}$ • Patrick Holman ${ }^{3}$ • Eric H. Yang ${ }^{1,4}$ (D) \\ Accepted: 8 January 2021 / Published online: 22 January 2021 \\ (C) The Author(s), under exclusive licence to Springer Science+Business Media, LLC part of Springer Nature 2021
}

\begin{abstract}
Purpose of Review Immunotherapies have demonstrated robust clinical efficacy in treating malignancies with increasing use and FDA approvals. We review the epidemiology, risk factors, diagnosis, and treatment of immunotherapy-associated cardiovascular toxicities.

Recent Findings Cardiotoxicity is reported in patients receiving immune checkpoint inhibitors (ICI) and chimeric antigen receptor (CAR) T cell therapies. The incidence of ICI-related cardiotoxicity is above $1 \%$ and includes myocarditis, pericardial disease, arrhythmia, acute coronary syndrome, and vasculitis. The incidence of CAR T cell-associated cardiotoxicities was shown to be as high as $26 \%$ and thought to be primarily mediated by cytokine release syndrome. The presentations of cardiotoxicities are variable but are associated with significant morbidity and mortality and benefit from prompt initiation of immunosuppressive therapy.

Summary There is increasing evidence for cardiotoxicities following cancer immunotherapy. Available evidence suggests that pretreatment evaluation, close monitoring, and early intervention may reduce cardiovascular morbidity and improve outcomes in the cancer immunotherapy population.
\end{abstract}

Keywords Cardiooncology $\cdot$ Immunotherapy $\cdot$ Immune checkpoint inhibitor $\cdot$ CAR-T cell therapy $\cdot$ Cardiotoxicity $\cdot$ Myocarditis

This article is part of the Topical Collection on Cardio-Oncology

Eric H. Yang

EHYang@mednet.ucla.edu

Ashley F. Stein-Merlob

asteinmerlob@mednet.ucla.edu

Michael V. Rothberg

mrothberg@mednet.ucla.edu

Patrick Holman

pholman@mednet.ucla.edu

1 Division of Cardiology, Department of Medicine, University of California, Los Angeles, Los Angeles, CA, USA

2 David Geffen School of Medicine, University of California, Los Angeles, Los Angeles, CA, USA

3 Department of Medicine, University of California, Los Angeles, Los Angeles, CA, USA

4 UCLA-Cardio-Oncology Program, Division of Cardiology, Department of Medicine, University of California, Los Angeles, 100 Medical Plaza, Suite 630, Los Angeles, CA 90095, USA

\section{Introduction}

Over the past decade, there has been remarkable progress in the treatment of cancer with the evolution from standard chemotherapeutic agents to targeted treatments and most recently to immunotherapy. Immunotherapies are a class of treatment modalities, including cytokines, antibodies, vaccines, and cellbased therapies that harness the capabilities of the immune system's antitumor response. Since the growth in immunotherapy, there has been an explosion of new, promising therapies. As of September 2017, there were 940 clinical and 1064 preclinical immunotherapies under investigation [1]. For immune checkpoint inhibitors (ICIs) alone, the percentage of eligible cancer patients has increased substantially from $1.54 \%$ in 2011 to $43.63 \%$ in 2018 [2]. In 2020, 1.8 million new cancer cases and over 600,000 cancer-related deaths are projected [3]; as adoption of immunotherapies increases, so too does the potential for rare immune related adverse reactions to these therapies.

Initial investigations of immunotherapy, starting decades ago, focused on the use of cytokines to nonspecifically upregulate the immune response, including IFN- $\alpha$ for hairy cell 
leukemia and IL-2 for renal cell carcinoma and metastatic melanoma. However, the use of these therapies was limited by systemic toxicity and upregulation of regulatory immune response [4]. Modulation of additional cytokines, including IL-10 and IL-6, is still under continued investigation [4].

The first major revolution in immunotherapy came with the development of ICIs. In the adaptive immune system, T cell activation is controlled by a delicate balance of costimulation and coinhibition pathways. Coinhibition pathway receptors on $\mathrm{T}$ cells, including cytotoxic $\mathrm{T}$ lymphocyte antigen 4 (CTLA4) or programmed cell death 1 (PD-1) receptors, can bind to ligands on antigen presenting cells, B7 and PD-L1 respectively. This coinhibition pathway receptor binding downregulates the immune response by decreasing $\mathrm{T}$ cell proliferation and migration or by increasing regulatory $\mathrm{T}$ cells. Tumor cells have adapted by overexpressing these ligands to evade the local immune response and proliferate unchecked [5-8]. Receptor antagonist monoclonal antibodies, later termed ICIs, block these pathways and reactivate the native $\mathrm{T}$ cell antitumor response. The first FDA-approved ICI treatment in 2011 was ipilimumab, a CTLA-4 antagonist, for treatment of metastatic melanoma [9]. There are now seven FDAapproved immune checkpoint inhibitors approved for use in a multitude of malignancies (Table 1). Although initially trialed in late stage disease, indications have rapidly evolved to include first-line or adjuvant indications. Despite the robust initial responses to these therapies, many patients develop resistance over time leading to the successful use of combination CTLA-4 and PD-1 antagonist treatments.

Chimeric antigen receptor (CAR) $\mathrm{T}$ cell therapy has emerged as a novel immunotherapy in which genetically engineered autologous cells are redirected to target surface antigens on cancer cells for destruction [10]. To date, three CD19-targeting CAR T cell therapies have gained FDA approval. Axicabtagene ciloleucel (Yescarta) is approved in the treatment of adult patients with relapsed or refractory large B cell lymphoma [11]. Tisagenlecleucel (Kymriah) is approved for patients with relapsed or refractory B cell precursor acute lymphoblastic leukemia (ALL) [12]. Most recently, brexucabtagene autoleucel (Tecartus) has gained approval in adults with relapsed or refractory mantle cell lymphoma [13].

With the proliferation of these novel therapies, there has been increased recognition of significant systemic adverse effects affecting every major organ system. Of particular concern, there have been increased reports of cardiovascular toxicities which, although rare, are potentially fatal complications of these life-prolonging immunotherapies.

\section{Immune Checkpoint Inhibitors}

Overall, up to 60 to $80 \%$ of patients treated with ICIs will experience at least one immune-related adverse event
(IRAE) during treatment [14]. Initially, cardiovascular IRAEs were thought to be very rare with early studies showing an incidence of 0.09 to $0.27 \%$ [15]. However, likely due to increased use of ICIs and increased recognition, more recent estimates of cardiovascular IRAEs reveal an incidence above 1 , ranging from 1.14 to over $5 \% \%[16 \bullet \bullet, 17,18 \bullet \bullet]$. The most recognized cardiovascular IRAE is myocarditis (79\%), but the presentation can also include arrhythmias, including atrial fibrillation $(30 \%)$, conduction disorders $(17 \%)$, or ventricular arrhythmias $(27 \%)$, pericardial disease, vasculitis, and takotsubo-like cardiomyopathy (14\%) [19]. Although still an uncommon entity compared to other immune related adverse events (IRAEs) such as colitis, dermatitis and pneumonitis, each with incidences of $>10 \%$, cardiovascular IRAEs are more likely to be severe and are associated with the highest mortality of all IRAEs at up to $50 \%$ [16••, 18••, 20•].

\section{Mechanism}

Immune checkpoints, including the CTLA-4 and PD-1 pathways, play an important role in suppressing $\mathrm{T}$ cell-mediated immune activation in the myocardium. In mouse models, loss of the PD-1 or CTLA-4 receptors induce infiltration of CD4+ and CD8+ T cells and development of a dilated cardiomyopathy [21-24]. Although the mechanism of ICI-induced cardiotoxicity has not been entirely elucidated, there are multiple prevailing theories including (1) immune dysregulation in the myocardium from overactivation of native T cells, (2) antitumor $\mathrm{T}$ cells cross reacting with antigens present in the myocardium, and (3) systemic immune response triggering cytokine release and local inflammation $[8,15,25 \bullet \bullet]$.

\section{Risk Factors}

Risk factors for development of cardiovascular IRAEs have been investigated in multiple retrospective studies; however, studies are limited due to the low incidence of cardiovascular IRAEs. The most established risk factor for development of ICI-associated cardiovascular IRAEs is treatment with combination ICI therapy, which conferred an almost 5-fold increased risk of ICI-associated myocarditis with combination compared monotherapy [15]. Additionally, combination therapy increases severity and mortality of ICI-associated myocarditis (mortality $65.6 \%$ in combination therapy vs $44.4 \%$ in monotherapy) $[15,16 \bullet \bullet, 20 \bullet]$. Between ICI agents, cardiovascular IRAEs are increased with CTLA-4 antagonists, like ipilimumab, compared to PD-1 or PD-1L inhibitors [15]. At this time, it is unclear if other targeted cancer therapies or radiation increase the risk of cardiovascular IRAEs.

Besides combination ICI therapy, few other risk factors have been identified. Registry data has shown a male predominance in cases of ICI-associated myocarditis, but this is likely confounded by an overrepresentation of males in ICI clinical 
Table 1 FDA approved indications of immune checkpoint inhibitors (ICIs) and recognized cardiotoxicities

\begin{tabular}{|c|c|c|c|c|c|}
\hline Drug (target) & FDA-approved indications (year) & FDA-labeled cardiotoxicity & Cardiac & Arrhythmia & Vascular \\
\hline Ipilimunab (CTLA-4) & Melanoma (2011) & $\begin{array}{l}\text { Pericarditis (incidence }<1 \% \text { ) } \\
\text { Myocarditis (incidence } 0.2 \% \text { ) }\end{array}$ & + & + & + \\
\hline Nivolumab (PD-1) & $\begin{array}{l}\text { Melanoma (2014), NSCLC (2015), RCC (2015), } \\
\text { classical Hodgkin lymphoma (2016), head and } \\
\text { neck SCC (2016), Urothelial carcinoma (2017), } \\
\text { HCC (2017), dMMR and MSI-h metastatic CRC } \\
\text { (2017), SCLC (2018), esophageal SCC (2020) }\end{array}$ & $\begin{array}{l}\text { Myocarditis (incidence }<1 \% \text { ), } \\
\text { ventricular arrhythmia }\end{array}$ & + & + & ++ \\
\hline Pembrolizumab (PD-1) & $\begin{array}{l}\text { Melanoma (2014), NSCLC (2015), head and neck } \\
\text { SCC (2016), Hodgkin lymphoma (2017), } \\
\text { urothelial carcinoma (2017), tumor mutational } \\
\text { burden-high solid tumors (2017), gastric or } \\
\text { gastroesophageal junction (2017), MSI-h or } \\
\text { dMMR solid tumors (2017), cervical cancer } \\
\text { (2018), Merkel cell carcinoma (2018), HCC } \\
\text { (2018), PMBCL (2018), RCC (2019), } \\
\text { endometrial carcinoma (2019), esophageal SCC } \\
\text { (2019), SCLC (2019), cutaneous SCC (2020), } \\
\text { MSI-h or dMMR CRC (2020) }\end{array}$ & Cardiac failure (incidence $0.4 \%$ ) & + & + & + \\
\hline Atezolizumab (PD-L1) & $\begin{array}{l}\text { Urothelial carcinoma (2016), NSCLC (2018), } \\
\text { SCLC (2019), PD-L1-positive triple-negative } \\
\text { breast cancer (2019), HCC (2020), melanoma } \\
(2020)\end{array}$ & Myocardial infarction & + & - & ++ \\
\hline Durvalumab (PD-L1) & $\begin{array}{l}\text { Urothelial carcinoma (2017), NSCLC (2018), } \\
\text { SCLC (2020) }\end{array}$ & Myocarditis (incidence $<1 \%$ & + & - & - \\
\hline Avelumab (PD-L1) & $\begin{array}{l}\text { Merkel cell carcinoma (2017), urothelial } \\
\text { carcinoma (2017), RCC (2020) }\end{array}$ & Myocarditis & + & - & - \\
\hline Cemiplimab (PD-1) & Advanced SCC (2018) & Myocarditis (case report) & NA & NA & NA \\
\hline
\end{tabular}

The breadth of FDA approved indications for unique malignancies has increased steadily since the initial FDA approval for each medication. All ICIs have FDA-labeled cardiotoxicities, but the frequency of the types of cardiotoxicity differs between therapies

$C R C$ colorectal cancer, $d M M R$ mismatch repair-deficient, $H C C$ hepatocellular carcinoma, $M S I-h$ high microsatellite instability, NSLC nonsmall cell lung cancer, $R C C$ renal cell carcinoma, SCC squamous cell carcinoma, SCLC small cell lung cancer. Toxicity ratings from Herrmann et al. 2020 [25••]: -not reported; +uncommon $(<1 \%)$; ++common $(1-10 \%)$; +++very common $(>10 \%)$

trials [20•]. A recent single center analysis of cancer patients receiving ICI suggested an association of myocarditis with an age of greater than 80 years, a history of heart failure, and/or acute coronary syndrome [26]. Future studies will be needed to better identify patients most at risk of developing cardiovascular IRAEs, particularly looking at prior or concurrent cardiotoxic cancer treatments, preexisting cardiovascular disease, or tumor-specific characteristics.

In the midst of the novel coronavirus disease-2019 (COVID-19) pandemic, COVID-19 myocarditis has been reported but is poorly defined [27]. Therefore, it is unclear what effect, if any, COVID-19 will have on the development of ICImyocarditis. In a retrospective case-control study by Awadalla et al. [28], a cohort of 101 ICI-associated myocarditis patients was compared to 201 controls on ICI therapy to determine the effect of the influenza vaccine as a mechanism to prevent concomitant viral myocarditis. The rate of vaccination was lower in patients who developed ICI-associated myocarditis compared to controls (25\% vs. $40 \%, p=0.01)$ [28]. Additionally, unvaccinated patients who developed ICIassociated myocarditis had 3-fold higher troponin elevation and 2-fold increased major adverse cardiac events compared to vaccinated ICI-associated myocarditis patients. The mechanism of this association is unclear, COVID-19 infections or future vaccines should be evaluated for an association with development of ICI-associated myocarditis.

\section{ICI-Associated Cardiotoxicities}

\section{Associated Myocarditis}

Presentation ICI-associated myocarditis is the most common cardiovascular IRAE and can have a broad range of presentations, from asymptomatic biomarker elevation to fulminant myocarditis with cardiogenic shock. The most common presenting symptoms include shortness of breath, palpitations, chest pain, and congestive heart failure [19]. The onset of ICI-related myocarditis most commonly occurs early in the course of ICI treatment with up to $80 \%$ occurring within the first 3 months of initiation of treatment, but studies have shown a wide range of onset $[16 \bullet \bullet, 18 \bullet \bullet, 19]$. In a multicenter registry of 35 patients, median onset was 34 days after initiation of ICI therapy (interquartile range: 21 to 75 days) [16••]. In a French database of ICI-associated myocarditis, onset occurred later 
with a median of 65 days (range 2-454 days) [19]. In the largest study of ICI-associated myocarditis by Moslehi et al. [18••], a retrospective review of 101 cases from VigiBase, the WHO database of safety reports, showed a median onset of 27 days (range of 5-155 days). Therefore, although ICI-associated myocarditis is most commonly seen after 2-3 treatments, the range of presentations requires a high index of suspicion both earlier and later in the course of treatment.

Diagnosis Initial workup of suspected ICI-associated myocarditis includes cardiac biomarkers, electrocardiogram (ECG), and echocardiogram with further consideration of advanced imaging techniques (Fig. 1). Endomyocardial biopsy (EMB) is still considered the gold standard for diagnosis of ICIassociated myocarditis, however, given the invasive nature and potential complications of EMB, diagnosis is often made based on clinical presentation, biomarker, and imaging results. In clinical research, the 2013 European Society of Cardiology guidelines for the clinical diagnosis of myocarditis have been adopted as a diagnostic criterion for ICI-associated myocarditis in the setting of active ICI therapy [29].

Biomarkers, particularly troponin and BNP, are sensitive, but lack specificity in ICI-associated myocarditis. In a multicenter case-control study, elevation of troponin and NTproBNP occurred in $94 \%$ and $66 \%$ of cases of ICIassociated myocarditis, respectively [16••]. The degree of troponin elevation has been associated with worse outcomes; specifically, troponin greater than $1.5 \mathrm{ng} / \mathrm{mL}$ at time of discharge has been associated with increased risk of major adverse cardiac events [16••].

ECG detects both isolated ICI-associated arrhythmia complications and myocarditis-associated rhythm disturbances. In a multicenter registry of 35 cases, ECG was abnormal in $89 \%$ of patients with ICI-associated myocarditis [16••]. The most common ECG abnormalities include atrial arrhythmias, ventricular arrhythmias, and conduction delays. Although ECG may be a sensitive screening tool for detection of ICIassociated myocarditis, due to the increased risk of arrhythmias independent of ICI-associated myocarditis it is has limited diagnostic value.

Echocardiography is an essential modality as first line evaluation for ICI-associated myocarditis. However, it is important to note that up to $50 \%$ of patients have normal left ventricular ejection fraction (LVEF) at time of evaluation [16••]. Global longitudinal stain (GLS) is a burgeoning tool in monitoring for subclinical cardiotoxicity with standard chemotherapy and shows promise in ICI-associated myocarditis. In a case-control study of 101 patients with ICI-associated myocarditis and 92 control patients on ICI therapy, both cohorts had similar baseline GLS, but there was a significant decrease in GLS in myocarditis $(14.1 \pm 2.8 \%)$ compared to controls $(20.5 \pm 1.9 \%, p<0.001)$. The reduction in GLS was more pronounced in patients with reduced LVEF $(12.3 \pm 2.7 \%)$ compared to preserved LVEF $(15.3 \pm 2.0 \%, p<0.001)$. Reduced GLS was strongly associated a 1.5-fold and 4.4-fold increased risk of MACE in reduced and preserved LVEF, respectively [30].

Cardiac MRI (CMR) has emerged as a crucial tool for the diagnosis of ICI-associated myocarditis due to the highresolution images and tissue characterization using late gadolinium enhancement (LGE) and T2-weighted STIR imaging of myocardial edema. Early studies of CMR in ICI-associated myocarditis showed relatively low sensitivity for CMR in detecting myocarditis. However, more recently in an international registry of 103 patients with ICI-associated myocarditis who underwent CMR, Zhang et al. [31•] provided the most complete view of cardiac MRI findings in ICI-associated myocarditis. Similar to echocardiographic studies, $61 \%$ of patients had a preserved LVEF of $>50 \%$. LGE was present in $48 \%$ of patients, of which $43 \%$ had preserved LVEF. The pattern of LGE was variable and included subendocardial/ transmural, subepicardial, midmyocardial, and diffuse
Fig. 1 Clinical management of immune checkpoint inhibitor (ICI)-associated myocarditis. Algorithm for management of patients with suspected myocarditis due to new symptoms or asymptomatic elevated troponin. $\mathrm{MCS}=$ mechanical circulatory support

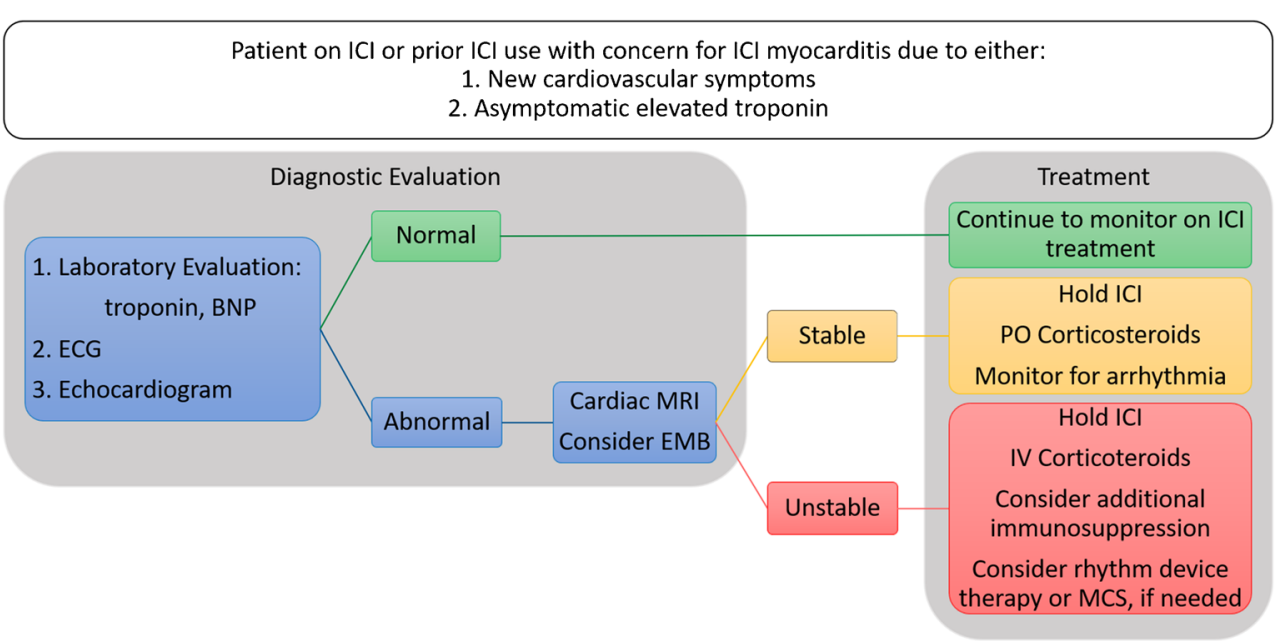


patterns. Myocardial edema was present in only $28 \%$ of patients. Of patients with LGE, there was no significant difference in demographics, presenting symptoms, outcomes, presenting ECG, or echocardiogram parameters. Patients with LGE had significantly higher initial troponin $\mathrm{T}(1.0 \mathrm{vs}$ $0.4 \mathrm{ng} / \mathrm{mL}, p=0.021)$. Most notably in this study, delayed CMR performed at least 4 days after admission was significantly associated with the presence of LGE (OR 9.35, $p<0.001$ ), with LGE present in only $21.6 \%$ of patients imaged within 4 days of admission but present in $72.0 \%$ of patients imaged on or after day 4 of admission [30]. This suggests that timing of CMR is important in the detection of ICI-associated myocarditis and may help explain limited sensitivity seen in earlier studies. T1 and T2 mapping may be promising CMR techniques to diagnose myocarditis, in which larger studies with standard protocols are warranted.

In addition to a comprehensive cardiovascular evaluation, patients should be evaluated for concomitant IRAE affecting other organ systems. One study showed that $42 \%$ of patients had concurrent severe IRAEs [18••]. The most commonly reported concomitant ICI-related toxicities are myositis and myasthenia gravis, occurring in up to $25 \%$ and $10 \%$ of patients with ICI myocarditis, respectively $[18 \bullet \bullet, 19]$.

Management ICI-associated myocarditis has a high rate of morbidity and mortality. In a study by Mahmood et al. [16*•] of 35 patients with ICI myocarditis, nearly one-half of all patients experienced a major adverse cardiac event including cardiovascular death $(n=6)$, cardiogenic shock $(n=3)$, cardiac arrest $(n=1)$, or complete heart block $(n=3)$. Factors that were associated with higher rate of MACE included higher admission, peak and discharge troponin $T$ values. Encouragingly, in patient survivors, complete recovery of EF was seen in approximately half of patients [18••].

At this time, no randomized controlled trials have determined the optimal treatment strategy for ICI-associated myocarditis, and current recommendations are based on expert opinion. The basic tenants of treatment of ICI-associated myocarditis are (1) stop ICI therapy, (2) immediate initiation of immunosuppression, and (3) supportive therapy. According to the most recent American Society for Clinical oncology (ASCO) guidelines, ICI therapy should be held for all ICIrelated cardiotoxicity and should be permanently discontinued for any cardiotoxicity more severe than grade I (asymptomatic biomarker elevations) [32]. There are case reports of rechallenging patients with ICIs; however, the risks of restarting therapy are unknown and thus would require a comprehensive multidisciplinary approach and informed discussion with the patient. Given the long half-life of ICIs (14.5 days for ipilimumab, 25.0 days for pembrolizumab, 26.7 days for nivolumab, and 27.0 days for atezolizumab), plasmapheresis has been used to remove the antibodies in severe cases $[25 \bullet \bullet$.
Corticosteroids are the mainstay of immunosuppressive treatment for ICI-associated myocarditis. Current ASCO guidelines recommend high-dose corticosteroids ( 1 to $2 \mathrm{mg} / \mathrm{kg}$ of prednisone) either oral or IV depending on severity of symptoms to be continued for 4-6 weeks based on clinical response [32]. A recent large, international multicenter registry of 126 ICI-associated myocarditis patients investigated the role of timing and dosage of corticosteroids [33•]. Patients who received early corticosteroids within $24 \mathrm{~h}$ of presentation had a lower rate of MACE compared to those receiving corticosteroids at $24-72 \mathrm{~h}$ or at $>72 \mathrm{~h}(7.0 \%, 34.3 \%$, and $85.1 \%$ respectively, $p<0.001)$. A variety of corticosteroids were used, including methylprednisolone $(76 \%)$, prednisone (20\%), hydrocortisone $(2 \%)$, and dexamethasone $(2 \%)$, and dosage was characterized according to methylprednisolone equivalents as low-dose $(<60 \mathrm{mg} /$ day, $16.7 \%)$, intermediate dose (60-500 mg/day, 43.7\%), and high dose (501-1000 mg/ day, $39.6 \%$ ). Occurrence of MACE was inversely related to the initial dose of corticosteroids. High dose corticosteroid was associated with $73 \%$ lower risk of MACE independent of timing of initiation (HR 0.27 [95\% CI, 0.09-0.84], $p=$ $0.024)\left[33^{\bullet}\right.$. These results suggest that early, high dose corticosteroid administration is associated with improved clinical outcomes. A future randomized controlled trial is needed to conclusively determine the optimal timing and dose of steroids in the treatment of ICI-associated myocarditis.

In addition to corticosteroids, adjuvant steroid-sparing agents have been used for treatment of fulminant ICIassociated myocarditis or cases refractory to corticosteroid therapy. Given similarities between the histopathology of ICI-associated myocarditis and cardiac allograft rejection, adjuvant treatments have been adapted from the antirejection drugs used in cardiac transplant, including mycophenolate, antithymocyte globulin (ATG), and infliximab [8, 16••, 32]. Use of infliximab is limited as it has been associated with worsening heart failure and is contraindicated in moderate to severe heart failure [5].

ICI-associated myocarditis causes significant MACE events including cardiogenic shock and life-threatening arrhythmias. Symptomatic patients should be evaluated with a multidisciplinary team of cardiology, oncology, and preferably cardiooncology specialists. Patients should be monitored on telemetry for development of arrhythmias due to potential need for temporary pacemakers or other acute interventions. Patients in cardiogenic shock should be monitored in a cardiac intensive care setting and may be evaluated for advanced mechanical circulatory support (MCS), including intraortic balloon pump, Impella (Abiomed, Danvers, MA) or extracorporeal membrane oxygenation (ECMO), if needed, as a bridge to recovery. No randomized controlled trial data has elucidated the management of cardiogenic shock, and the decision to escalate to MCS depends on many factors 
including the stage of malignancy, cancer prognosis and the patient's goals of care.

ICI-Associated Pericardial Disease ICI-associated pericardial disease can present as pericarditis, perimyocarditis, or pericardial effusion. Pericarditis is diagnosed with typical pericardial pain, ECG changes with diffuse ST elevation and PR depression or new pericardial effusion. Patients with suspected pericardial disease should undergo workup with troponin, ECG, and echocardiogram. In a large WHO registry, the onset of pericardial disorders occurred with a median onset of 30 days (IQR: 8.4-90 days). ICI-associated pericardial disorders were most commonly reported in patients with lung cancer [20•]. In a retrospective study of patients with stage IIB/IV NSCLC treated with ICIs at a single center over 2 years, review of echocardiograms revealed an increased incidence of pericardial effusions compared to standard chemotherapy (3.3\% vs $1.6 \%$ respectively) without concomitant myocarditis as determined by troponin elevation [34]. Pericardial effusions have been complicated by cardiac tamponade and mortality is $21.1 \%$ [20•]. Optimal treatment strategies, including the use of steroids, nonsteroidal antiinflammatories, and colchicine, are unknown and require further study.

ICl-Associated Arrhythmia Various arrhythmias have been described in the setting of ICI therapy including conduction delays, ventricular arrhythmias, and atrial fibrillation [19, 35]. Conduction disease and ventricular arrhythmias are thought to be primarily driven by inflammation, either locally in the ventricle or His-Purkinje system or systemic inflammation. Atrial fibrillation may be due to myocarditis, pericarditis, systemic inflammation, or secondary to other IAER such as thyroiditis $\left[7,20 \bullet, 25 \bullet^{\bullet}\right]$. Importantly, arrhythmic complications have been implicated as a major driver of mortality and sudden cardiac death [19]. Patients with symptoms of palpitations or syncope should undergo 12-lead ECG or ambulatory ECG monitoring to evaluate for arrhythmias.

ICl-Associated Acute Coronary Syndrome A metaanalysis of 22 ICI trials in lung cancer showed $1 \%$ incidence of ICIassociated myocardial infarction [17]. In addition, a single center study of 3326 patients receiving ICI revealed an $7 \%$ incidence of myocardial infarction - and a similar incidence of stroke of note - over a mean follow-up of 16 months [26]. It is unclear if these reports of myocardial infarction represent true atherosclerotic plaque rupture or another complication of ICI therapy including vasculitis, coronary vasospasm, or myocarditis. However, there is emerging evidence of a link with $\mathrm{T}$ cell activity and atherosclerotic plaque stability; given PD-1 levels are elevated in plaque T cells, ICI therapy (e.g. PD-1 inhibition) may theoretically activate these $\mathrm{T}$ cells and worsen atherosclerotic disease with cardiac ischemic events as a consequence [36]. Patients with symptoms and ECG findings consistent with myocardial infarction should be treated with standard of care and undergo coronary angiography with possible percutaneous coronary intervention, if needed.

ICI-Associated Vasculitis Vasculitis has been reported in increased frequency in patients on ICI therapy, most commonly polymyalgia rheumatica and temporal arteritis [20•]. The onset of presentation is relatively delayed compared to other cardiovascular IRAEs with a median onset of 55 days (IQR: 2-98 days) [20•]. Although the mortality is lower than other cardiovascular IRAEs at $6.1 \%$, there was impaired vision in $27.8 \%$ of temporal arteritis cases [20॰]. Due to the high risk of vision loss, treatment of temporal arteritis should include stopping ICI administration and initiating immunosuppression with corticosteroid dosing according to current guidelines of treatment of temporal arteritis [5].

\section{Chimeric Antigen Receptor T Cell Therapy}

While CAR T cell therapies have demonstrated robust clinical efficacy in hematological malignancies [37], an array of adverse cardiovascular outcomes have been identified with the development of high-grade cytokine release syndrome (CRS) recognized as a key mechanistic driver [25]. Available data on CD19-targeting CAR T cell therapies have found varying incidences of cardiotoxicity. In a 145 CAR T cell adult patient cohort, 31 patients experienced major adverse cardiovascular events (21\%) with grade 3-4 CRS conferring greater risk [38••]. However, a cohort of 137 adult patients undergoing CAR T cell for diffuse large B cell lymphoma (DLBCL), including transformed follicular lymphoma and multiple myeloma, observed a lower incidence, with 17 cardiovascular events $(12 \%)$, all of which coincided with grade $\geq 2$ CRS, and included 6 cardiovascular deaths [39••]. In a 98 patient exclusively pediatric cohort, the primary outcome of hypotension requiring vasoactive medication occurred in 24 patients (25\%) [40••]. The observed cardiovascular adverse outcomes following CAR T cell to date encompass arrhythmias (including atrial fibrillation, supraventricular tachycardia (SVT), atrioventricular nodal blocks, and prolonged QT), tachycardia, hypotension, heart failure, decreased LVEF, and in rare cases cardiac arrest [25••, 37].

\section{Mechanism}

The mechanisms of cardiotoxicity for CD19-targeting CAR-T cell therapies are thought to be primarily mediated by the systemic inflammation of CRS [41]. In retrospective analyses of patients receiving CAR-T therapy, the degree of CRS has been strongly associated with the development of adverse cardiovascular outcomes $[38 \bullet \bullet, 39]$. Upon recognition of the tumor antigen domain, the activated CAR-T cells release 
numerous cytokines including IFN $\gamma$, IL-1, IL-2RA, TNF $\alpha$, and the most well-studied mediator, IL-6 [42]. This activation serves as the foundation of CAR-T cell therapy by eliciting cytotoxic responses toward CD19+ tumor cells; in fact, most patients who respond to CAR-T therapy develop some degree of CRS [43]. However, CRS also activates prostaglandins and can cause fever, myalgias, fatigue, hypotension, tachycardia, capillary leak, and multiple organ toxicities including the central nervous system and cardiovascular system [41, 42]. Additionally, as future CAR-T cell therapies are developed with alternative targets, there is increased risk for direct "offtarget, off-tumor" cardiotoxicity such as that observed in an early engineered $\mathrm{T}$ cell receptor targeting melanomaassociated antigen 3 (MAGEA3) that resulted in fatal cardiogenic shock due to cross-reactivity with titin causing infiltrative myocardial damage $[44,45]$.

The clinical constellation of CAR T cell-associated cardiotoxicity can mirror that of sepsis, with hypotension, tachycardia, and decreased LVEF occurring in both settings, but they are mechanistically distinct. The cytokine profile of septic cardiomyopathy is more strongly associated with TNF $\alpha$ and IL- $1 \beta$ compared to the marked IL- 6 elevations of CAR T cell inflammatory cardiotoxicity [25••]. Levels of IL-6 rise dramatically following CAR T cell therapy [46], and IL-6 has a variety of roles in the development of CRS, including the recruitment of additional $T$ cells via upregulated expression of attractive chemokines and the facilitation of lymphocyte differentiation [47]. The administration of tocilizumab, an inhibitor of IL-6, has been shown to impede the progression of CRS [48]. Moreover, the overlapping but mechanistically distinct pathophysiology of septic cardiomyopathy is marked by complement system and nitric oxide dysregulation secondary to bacterial endotoxin exposure [49].

\section{Risk Factors}

Retrospective observational studies of adult and pediatric CAR $T$ cell therapy populations have provided associative evidence to identify risk factors for cardiotoxicity in CAR T cell therapy. In a single-site study of pediatric ALL patients, pretreatment high tumor cell burden (blast $>25 \%$ ) was associated with a higher incidence of hypotension requiring inotropic support. Preexisting cardiomyopathy, past total body irradiation, and anthracycline dose were not associated with increased incidence of hypotension [40,50]. The development of high grade CRS after infusion is associated with higher incidence of adverse cardiovascular outcomes following CAR T cell infusion $[38 \bullet \bullet, 39 \bullet \cdot]$.

CAR T cell therapy is specifically indicated for relapsed or refractory malignancies; therefore, patients have typically been previously treated with chemotherapeutic or irradiative regimens. Past treatment with medications carrying known cardiovascular risk profiles, including TKI's, anthracyclines, alkylating agents, antimetabolites, microtubule inhibitors, and various targeted agents, increase cardiovascular toxicity risk in the context of CAR T cell therapy [25••]. Baseline use of insulin, statin, aspirin, and higher creatinine are also associated with adverse cardiovascular outcomes, perhaps reflecting an increased baseline cardiovascular risk profile before CAR T cell treatment [38••]. Larger scale, multiinstitutional studies are needed to fully characterize the relative contributions of these potential risk factors.

Currently, there are no formal guidelines for risk stratification or preCAR T cell therapy cardiovascular evaluation. In patients with a history of cardiovascular symptoms, abnormal ECG or echocardiography, pre-existing CVD, or impaired exercise tolerance, one proposed evaluation involves a cardiooncology consultation with diagnostics tailored to a patient's risk. For patients with cardiovascular risk factors or prior cardiotoxic therapies (i.e., anthracyclines), consider baseline echocardiogram and ischemic evaluation to allow optimization of pretreatment cardiovascular status [51]. Currently, there are no absolute contraindications for CAR T cell therapy, but the high incidence of cardiovascular sequelae merit thorough pretreatment evaluation.

\section{Presentation and Diagnosis}

The presentation of CAR T cell therapy-associated cardiotoxicity can include hypotension, tachycardia, arrhythmias, or new left ventricular systolic dysfunction, and can occur at varying time points after initiation of CAR $\mathrm{T}$ cell therapy. Troponin elevations in adults receiving CAR T cell therapy had an interquartile range of 6 to 31 days after infusion [39••] and a separate cohort of adults receiving CAR T cell had an interquartile range of major adverse cardiovascular events of 6 to 151 days (Table 2) [38••].

De novo CAR T cell-associated arrhythmias have been consistently observed and manifest most commonly as atrial fibrillation or atrial flutter [25••]. In a 145 adult CAR T cell patient cohort, the overall incidence of arrhythmia was 14 episodes in 13 patients $(9.0 \%)$, including atrial fibrillation $(n=12)$, SVT $(n=1)$ and nonsustained ventricular tachycardia $(n=1)$ [38••]. In another study of 137 adult CAR T cell therapy patients, new arrhythmias occurred in 7 patients (5.1\%), including atrial fibrillation, atrial flutter, and SVT [39••]. Though rare, QTc prolongation has also been noted in multiple studies [39॰•, 52]. Given the prevalence of these arrhythmias, ECG monitoring following infusion may help identify potentially life-threatening rhythms.

Additionally, new systolic dysfunction, acute coronary syndrome, ischemic stroke, and cardiovascular-related death have been observed following CAR T cell therapy across studies in association with high grade CRS, although frequen-

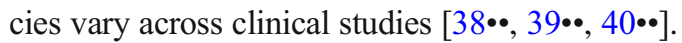


Table 2 Frequencies of adverse cardiovascular events in CAR T cell therapy trials

\begin{tabular}{|c|c|c|c|c|c|}
\hline Study & $\begin{array}{l}\text { Population } \\
\text { (indication) } \\
(n)\end{array}$ & Therapy & $\begin{array}{l}\text { \# Patients with adverse } \\
\text { CV events }[n(\%)]\end{array}$ & Adverse CV event & $\begin{array}{l}\text { \# Patients } \\
{[n(\%)]}\end{array}$ \\
\hline \multirow[t]{5}{*}{ Lefebvre et al. 2020 [38] } & \multirow{5}{*}{$\begin{array}{l}\text { Adults (DLBCL, } \\
\text { ALL, CLL) } \\
(n=145)\end{array}$} & \multirow[t]{5}{*}{ CD19-directed CAR T } & \multirow[t]{5}{*}{$38(26 \%)$} & Cardiovascular death & $2(1.4 \%)$ \\
\hline & & & & Symptomatic HF & $21(15 \%)$ \\
\hline & & & & Nonfatal ACS & $2(1.4 \%)$ \\
\hline & & & & New atrial fibrillation & $11(7.6 \%)$ \\
\hline & & & & New other arrhythmia & $2(1.4 \%)$ \\
\hline \multirow[t]{4}{*}{ Alvi et al. 2019 [39] } & \multirow{4}{*}{$\begin{array}{l}\text { Adults (DLBCL, } \\
\text { MM, transformed } \\
\text { follicular, other }) \\
(n=137)\end{array}$} & \multirow{4}{*}{$\begin{array}{l}\text { CD19-directed CAR T } \\
\text { (or investigational } \\
\text { CAR T) }\end{array}$} & \multirow[t]{4}{*}{$21(15 \%)$} & Cardiovascular death & $6(11 \%)$ \\
\hline & & & & New onset HF & $6(11 \%)$ \\
\hline & & & & HF decompensation & $2(3.6 \%)$ \\
\hline & & & & Any new arrhythmia & $7(12 \%)$ \\
\hline \multirow[t]{3}{*}{ Burstein et al. 2018 [40] } & \multirow[t]{3}{*}{$\begin{array}{l}\text { Pediatrics (ALL) } \\
\quad(2-27 \text { yo })(n=93)\end{array}$} & \multirow[t]{3}{*}{ CD19-directed CAR T } & \multirow[t]{3}{*}{$24(26 \%)$} & $\begin{array}{l}\text { Hypotension requiring } \\
\text { pressors (no echocardiography) }\end{array}$ & $4(4 \%)$ \\
\hline & & & & $\begin{array}{l}\text { Hypotension requiring pressors } \\
\text { (normal echocardiography) }\end{array}$ & $10(10 \%)$ \\
\hline & & & & $\begin{array}{l}\text { Hypotension requiring pressors } \\
\text { (echocardiographic dysfunction) }\end{array}$ & $10(10 \%)$ \\
\hline
\end{tabular}

Summary of clinical trials of CD-19 directed CAR T cell therapy trials showing the frequency and type of cardiovascular toxicity $A C S$ acute coronary syndrome, $A L L$ acute lymphoblastic leukemia, $C L L$ chronic lymphocytic leukemia, $D L B C L$ diffuse large $\mathrm{B}$ cell lymphoma, $H F$ heart failure

\section{Management of CRS and CAR T Cell-Related Cardiotoxicity}

A strong relationship between CRS and CAR T cell-related

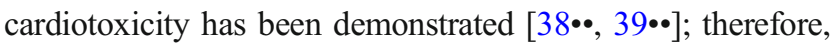
clinical recognition of CRS and the mitigation of CRS progression to higher grades are essential to lowering the risk of cardiotoxicity. Several distinct but overlapping grading schema to quantify CRS have been developed [10,53-58]. The definition of grade I CRS includes the development of fever $\geq$ $38.0^{\circ} \mathrm{C}$. In the ASTCT consensus criteria, hypotension responsive to IV fluids or one vasopressor constitutes grade II CRS, hypoxia requiring high-flow oxygen constitutes grade III CRS, and hypoxia requiring positive pressure ventilation or hypotension requiring multiple vasopressors constitutes grade III CRS [54]. Differences between grading definitions in multiple guidelines adds to the complexity of comparing outcomes in various clinical trials. Consolidation of these guidelines would allow for increased comparability between cardiotoxicity studies and the future development of robust clinical guidelines to improve treatment.

Preventing the progression of CRS improves overall cardiovascular outcomes in CAR T cell patients. Tocilizumab is a humanized monoclonal antibody inhibitor of IL-6 primarily used in the treatment of rheumatoid arthritis, which has been shown to prevent the progression of CRS [10]. In a study of 137 adult CAR T cell patients, a shorter elapsed time between the onset of CRS and the administration of tocilizumab was associated with lower rates of adverse cardiovascular events, suggesting that the alleviation of CRS may be beneficial in staving off inflammatory-mediated cardiotoxicities [39••]. In contrast to corticosteroids, which can be a necessary intervention in the setting of severe systemic inflammation, tocilizumab has not been found to negatively impact the efficacy of CAR T cell therapy itself. In a trial of preemptive tocilizumab in CRS following CD19-targeting CAR T cell therapies, the early administration of tocilizumab with or without corticosteroids in relapsed or refractory ALL prevented the progression of mild CRS without impacting rates of minimal residual disease negative complete remission rates or the persistence of CAR T cell populations compared to subjects not receiving tocilizumab [48]. Further study is required to determine the precise severity of CAR $\mathrm{T}$ cell related CRS that warrants tocilizumab versus more aggressive corticosteroid interventions. Currently, evidence suggests that tocilizumab is an effective means of preventing CRS progression beyond grade 2 CRS, and that high dose corticosteroids should be reserved for antilL6-refractory, life-threatening cases of CRS [58].

\section{Conclusion}

With the increased use of both ICI and CAR-T cell therapy, immunotherapy associated cardiotoxicity will become increasingly important in general cardiovascular care. While still considered rare toxicities of these treatments, each toxicity confers significant risk of MACE and mortality. Treatment 
of these toxicities requires prompt recognition and treatment to ensure optimal outcomes. In this burgeoning field, there are still many unmet needs to better understand the optimal management of these patients. International registries are vital to understanding the true incidence, risk factors, and prognosis of both ICI-associated and CAR-T cell-associated cardiotoxicities. With better understanding of risk factors, there should be standardized guidelines for high risk patient cardiovascular evaluation prior to and monitoring after initiation of therapy. With increased multinational collaborations, randomized controlled trials are needed to elucidate the optimal treatment strategies, particularly in the use of adjuvant immunosuppressive agents in ICI-associated myocarditis. Finally, as new immunotherapies are developed and adopted into clinical practice, it will be important to include cardiovascular surveillance in clinical trials for early detection of potential new cardiovascular toxicities.

\section{Compliance with Ethical Standards}

Conflict of Interest The authors declare that they have no relevant conflicts of interest.

Human and Animal Rights and Informed Consent This article does not contain any studies with human or animal subjects performed by any of the authors.

\section{References}

Papers of particular interest, published recently, have been highlighted as:

- Of importance

•- Of major importance

1. Zaha VG, Meijers WC, Moslehi J. Cardio-immuno-oncology. Circulation. 2020;141(2):87-9. https://doi.org/10.1161/ CIRCULATIONAHA.119.042276.

2. Haslam A, Prasad V. Estimation of the percentage of US patients with cancer who are eligible for and respond to checkpoint inhibitor immunotherapy drugs. JAMA Netw Open. 2019;2(5):e192535. https://doi.org/10.1001/jamanetworkopen.2019.2535.

3. Siegel RL, Miller KD, Jemal A. Cancer statistics, 2020. CA Cancer J Clin. 2020;70(1):7-30. https://doi.org/10.3322/caac.21590.

4. Naing A, Hajjar J, Immunotherapy. 3 ed., vol 1244. Springer International Publishing; 2020. https://doi.org/10.1007/978-3-03041008-7.

5. Hu J-R, Florido R, Lipson EJ, Naidoo J, Ardehali R, Tocchetti CG, et al. Cardiovascular toxicities associated with immune checkpoint inhibitors. Cardiovasc Res. 2019;115(5):854-68. https://doi.org/10. 1093/cvr/cvz026.

6. Tajiri K, Ieda M. Cardiac complications in immune checkpoint inhibition therapy. Front Cardiovasc Med. 2019;6:3. https://doi. org/10.3389/fcvm.2019.00003.

7. Lyon AR, Yousaf N, Battisti NML, Moslehi J, Larkin J. Immune checkpoint inhibitors and cardiovascular toxicity. Lancet Oncol. 2018;19(9):e447-e58. https://doi.org/10.1016/S1470-2045(18) $30457-1$.
8. Khunger A, Battel L, Wadhawan A, More A, et al. New insights into mechanisms of immune checkpoint inhibitor-induced cardiovascular toxicity. Curr Oncol Rep. 2020;22(65). https://doi.org/10. 1007/s11912-020-00925-8.

9. Hodi FS, O'Day SJ, McDermott DF, Weber RW, Sosman JA, Haanen JB, et al. Improved survival with ipilimumab in patients with metastatic melanoma. N Engl J Med. 2010;363(8):711-23. https://doi.org/10.1056/nejmoa1003466.

10. Neelapu SS, Tummala S, Kebriaei P, Wierda W, Gutierrez C, Locke FL, et al. Chimeric antigen receptor T-cell therapy - assessment and management of toxicities. Nat Rev Clin Oncol. 2018;15(1):47-62. https://doi.org/10.1038/nrclinonc.2017.148.

11. Schuster SJ, Bishop MR, Tam CS, Waller EK, Borchmann P, McGuirk JP, et al. Tisagenlecleucel in adult relapsed or refractory diffuse large B-cell lymphoma. N Engl J Med. 2019;380(1):45-56. https://doi.org/10.1056/nejmoa1804980.

12. Maude SL, Laetsch TW, Buechner J, Rives S, Boyer M, Bittencourt $\mathrm{H}$, et al. Tisagenlecleucel in children and young adults with B-cell lymphoblastic leukemia. N Engl J Med. 2018;378(5):439-48. https://doi.org/10.1056/nejmoa1709866.

13. Wang M, Munoz J, Goy A, Locke FL, Jacobson CA, Hill BT, et al. KTE-X19 CAR T-cell therapy in relapsed or refractory mantle-cell lymphoma. N Engl J Med. 2020;382(14):1331-42. https://doi.org/ 10.1056/nejmoa1914347.

14. Michot JM, Bigenwald C, Champiat S, Collins M, Carbonnel F, Postel-Vinay $\mathrm{S}$, et al. Immune-related adverse events with immune checkpoint blockade: a comprehensive review. Eur J Cancer. 2016;54:139-48. https://doi.org/10.1016/j.ejca.2015.11.016.

15. Johnson DB, Balko JM, Compton ML, Chalkias S, Gorham J, Xu $\mathrm{Y}$, et al. Fulminant myocarditis with combination immune checkpoint blockade. N Engl J Med. 2016;375(18):1749-55. https://doi. org/10.1056/nejmoa1609214.

16.• Mahmood SS, Fradley MG, Cohen JV, Nohria A, Reynolds KL, Heinzerling LM, et al. Myocarditis in patients treated with immune checkpoint inhibitors. J Am Coll Cardiol. 2018;71(16):1755-64. https://doi.org/10.1016/j.jacc.2018.02.037 Multicenter registry of ICI-associated myocarditis with 35 patients to evaluate the onset, risk factors and outcomes in ICI-associated myocarditis.

17. Hu Y-B, Zhang Q, Li H-J, Michot JM, Liu H-B, Zhan P, et al. Evaluation of rare but severe immune related adverse effects in PD-1 and PD-L1 inhibitors in non-small cell lung cancer: a metaanalysis. Translat Lung Cancer Res. 2017;6(S1):S8-S20. https:// doi.org/10.21037/tlcr.2017.12.10.

18.• Moslehi JJ, Salem J-E, Sosman JA, Lebrun-Vignes B, Johnson DB. Increased reporting of fatal immune checkpoint inhibitor-associated myocarditis. Lancet. 2018;391(10124):933. https://doi.org/10. 1016/S0140-6736(18)30533-6 Retrospective study of WHO database of $\mathbf{1 0 1}$ cases of ICI-associated myocarditis to evaluate the incidence and associated risk factors for ICI-associated myocarditis.

19. Escudier M, Cautela J, Malissen N, Ancedy Y, Orabona M, Pinto J, et al. Clinical features, management, and outcomes of immune checkpoint inhibitor-related Cardiotoxicity. Circulation. 2017;136(21):2085-7. https://doi.org/10.1161/circulationaha.117. 030571 .

20. Salem J-E, Manouchehri A, Moey M, Lebrun-Vignes B, Bastarache L, Pariente A, et al. Cardiovascular toxicities associated with immune checkpoint inhibitors: an observational, retrospective, pharmacovigilance study. Lancet Oncol. 2018;19(12):157989. https://doi.org/10.1016/s1470-2045(18)30608-9 Pharmacovigilance study using the WHO drug safety reporting database to compare incidence of cardiotoxicity in ICIs. They identified increased incidence of myocarditis, pericardial disease and vasculitis with significant associated morbidity and mortality. 
21. Nishimura H, Okazaki T, Tanaka Y, Nakatani K, Hara M, Matsumori A, et al. Autoimmune dilated cardiomyopathy in PD1 receptor-deficient mice. Science. 2001;291(5502):319-22. https://doi.org/10.1126/science.291.5502.319.

22. Wang J, Okazaki I-M, Yoshida T, Chikuma S, Kato Y, Nakaki F, et al. PD-1 deficiency results in the development of fatal myocarditis in MRL mice. Int Immunol. 2010;22(6):443-52. https://doi. org/10.1093/intimm/dxq026.

23. Tivol EA, Borriello F, Schweitzer AN, Lynch WP, Bluestone JA, Sharpe AH. Loss of CTLA-4 leads to massive lymphoproliferation and fatal multiorgan tissue destruction, revealing a critical negative regulatory role of CTLA-4. Immunity. 1995;3(5):541-7. https:// doi.org/10.1016/1074-7613(95)90125-6.

24. Lichtman AH. The heart of the matter: protection of the myocardium from T cells. J Autoimmun. 2013;45:90-6. https://doi.org/10. 1016/j.jaut.2013.05.004.

25.• Herrmann J. Adverse cardiac effects of cancer therapies: cardiotoxicity and arrhythmia. Nat Rev Cardiol. 2020;17(8):474 502. https://doi.org/10.1038/s41569-020-0348-1 This is a comprehensive review of the adverse cardiac effects of all cancer therapies, including immunotherapies.

26. Oren O, Yang EH, Molina JR, Bailey KR, Blumenthal RS, Kopecky SL. Cardiovascular health and outcomes in cancer patients receiving immune checkpoint inhibitors. Am J Cardiol. 2020;125:1920-6.

27. Puntmann VO, Carerj ML, Wieters I, Fahim M, Arendt C, Hoffmann J, et al. Outcomes of cardiovascular magnetic resonance imaging in patients recently recovered from coronavirus disease 2019 (COVID-19). JAMA Cardiol. 2020;5:1265-73. https://doi. org/10.1001/jamacardio.2020.3557.

28. Awadalla M, Golden DLA, Mahmood SS, Alvi RM, Mercaldo ND, Hassan MZO, et al. Influenza vaccination and myocarditis among patients receiving immune checkpoint inhibitors. J ImmunoTherapy Cancer. 2019;7(1). https://doi.org/10.1186/ s40425-019-0535-y.

29. Caforio AL, Pankuweit S, Arbustini E, Basso C, Gimeno-Blanes J, Felix SB, et al. Current state of knowledge on aetiology, diagnosis, management, and therapy of myocarditis: a position statement of the European Society of Cardiology Working Group on Myocardial and Pericardial Diseases. Eur Heart J. 2013;34(33):2636-48, 48a48d. https://doi.org/10.1093/eurheartj/eht210.

30. Awadalla M, Mahmood SS, Groarke JD, Hassan MZO, Nohria A, Rokicki A, et al. Global longitudinal strain and cardiac events in patients with immune checkpoint inhibitor-related myocarditis. J Am Coll Cardiol. 2020;75(5):467-78. https://doi.org/10.1016/j. jacc.2019.11.049.

31. Zhang L, Awadalla M, Mahmood SS, Nohria A, Hassan MZO, Thuny F, et al. Cardiovascular magnetic resonance in immune checkpoint inhibitor-associated myocarditis. Eur Heart J. 2020;4: ehaa051. https://doi.org/10.1093/eurheartj/ehaa051 International multicenter registry of 103 patients with ICI-associated myocarditis who underwent cardiac MRI that characterized the heterogeneity of cardiac MRI findings seen in ICI-myocarditis, including presence of late gadolinium enhancement and myocardial edema. There was relatively low sensitivity of cardiac MRI in detection of ICI-associated myocarditis, but sensitivity improves with delayed imaging.

32. Brahmer JR, Lacchetti C, Schneider BJ, Atkins MB, Brassil KJ, Caterino JM, et al. Management of immune-related adverse events in patients treated with immune checkpoint inhibitor therapy: American Society of Clinical Oncology clinical practice guideline. JCO. 2018;36(17):1714-68. https://doi.org/10.1200/JCO.2017.77. 6385.

33. Zhang L, Zlotoff DA, Awadalla M, Mahmood SS, Nohria A, Hassan MZO, et al. Major adverse cardiovascular events and the timing and dose of corticosteroids in immune checkpoint inhibitor- associated myocarditis. Circulation. 2020;141(24):2031-4. https:// doi.org/10.1161/CIRCULATIONAHA.119.044703 International multicenter registry of $\mathbf{1 0 3}$ patients with ICI-associated myocarditis who underwent cardiac MRI that characterized the heterogeneity of cardiac MRI findings seen in ICI-myocarditis, including presence of late gadolinium enhancement and myocardial edema. There was relatively low sensitivity of cardiac MRI in detection of ICI-associated myocarditis, but sensitivity improves with delayed imaging.

34. Canale ML, Camerini A, Casolo G, Lilli A, Bisceglia I, Parrini I, et al. Incidence of pericardial effusion in patients with advanced non-small cell lung cancer receiving immunotherapy. Adv Ther. 2020;37:3178-84. https://doi.org/10.1007/s12325-020-01386-y.

35. Mir H, Alhussein M, Alrashidi S, Alzayer H, Alshatti A, Valettas N, et al. Cardiac complications associated with checkpoint inhibition: a systematic review of the literature in an important emerging area. Can J Cardiol. 2018;34(8):1059-68. https://doi.org/10.1016/j.cjca. 2018.03.012.

36. Fernandez DM, Rahman AH, Fernandez NF, Chudnovskiy A, Amir EAD, Amadori L, et al. Single-cell immune landscape of human atherosclerotic plaques. Nat Med. 2019;25(1):1576-88.

37. Neelapu SS. Managing the toxicities of CAR T-cell therapy. Hematol Oncol. 2019;37(S1):48-52. https://doi.org/10.1002/hon. 2595.

38.• Lefebvre B, Kang Y, Smith AM, Frey NV, Carver JR, ScherrerCrosbie M. Cardiovascular effects of CAR T cell therapy. JACC: CardioOncol. 2020;2(2):193-203. https://doi.org/10.1016/j.jaccao. 2020.04.012 Retrospective, single center study of $\mathbf{1 4 5}$ adult patients undergoing CAR T-cell therapy to evaluate for indidence and risk factors for cardiotoxicity. This study found increased risk for MACE events with CAR T-therapy. There were associations between cardiotoxicity and CRS, baseline renal function and baseline cardiovascular status.

39.• Alvi RM, Frigault MJ, Fradley MG, Jain MD, Mahmood SS, Awadalla M, et al. Cardiovascular events among adults treated with chimeric antigen receptor T-cells (CAR-T). J Am Coll Cardiol. 2019;74(25):3099-108. https://doi.org/10.1016/j.jacc.2019.10.038 Retrospective, single center study of 137 adult patients undergoing CAR T-cell therapy evaluating the incidence of cardiovascular toxicity. In particular, this study showed an incidence of $12 \%$ of all patients undergoing therapy with significant association with development of cytokine release syndrome and improved outcome with tocilizumab.

40.• Burstein DS, Maude S, Grupp S, Griffis H, Rossano J, Lin K. Cardiac profile of chimeric antigen receptor T cell therapy in children: a single-institution experience. Biol Blood Marrow Transplant. 2018;24(8):1590-5. https://doi.org/10.1016/j.bbmt. 2018.05.014 Retrospective, single center pediatric study of $\mathbf{9 8}$ patients undergoing CAR T-cell therapy. This study found an association between development of hypotension requiring inotropic intervention and pre-existing cardiac dysfunction and pre-treatment blast count.

41. Asnani A. Cardiotoxicity of immunotherapy: incidence, diagnosis, and management. Curr Oncol Rep. 2018;20(6):1. https://doi.org/10. 1007/s11912-018-0690-1.

42. Oved JH, Barrett DM, Teachey DT. Cellular therapy: immunerelated complications. Immunol Rev. 2019;290(1):114-26. https:// doi.org/10.1111/imr.12768

43. Leick MB, Maus MV. Toxicities associated with immunotherapies for hematologic malignancies. Best Pract Res Clin Haematol. 2018;31(2):158-65. https://doi.org/10.1016/j.beha.2018.03.004.

44. Linette GP, Stadtmauer EA, Maus MV, Rapoport AP, Levine BL, Emery L, et al. Cardiovascular toxicity and titin cross-reactivity of affinity-enhanced $\mathrm{T}$ cells in myeloma and melanoma. Blood. 2013;122(6):863-71. https://doi.org/10.1182/blood-2013-03490565. 
45. Cameron BJ, Gerry AB, Dukes J, Harper JV, Kannan V, Bianchi FC, et al. Identification of a Titin-derived HLA-A1-presented peptide as a cross-reactive target for engineered MAGE A3-directed T cells. Sci Transl Med. 2013;5(197):197ra03-ra1. https://doi.org/10. 1126/scitranslmed.3006034.

46. Titov A, Petukhov A, Staliarova A, Motorin D, Bulatov E, Shuvalov O, et al. The biological basis and clinical symptoms of CAR-T therapy-associated toxicites. Cell Death Dis. 2018;9(9). https://doi.org/10.1038/s41419-018-0918-X.

47. Scheller J, Chalaris A, Schmidt-Arras D, Rose-John S. The pro- and anti-inflammatory properties of the cytokine interleukin-6. Biochim Biophys Acta (BBA). 2011;1813(5):878-88. https://doi.org/10. 1016/j.bbamcr.2011.01.034

48. Gardner RA, Ceppi F, Rivers J, Annesley C, Summers C, Taraseviciute A, et al. Preemptive mitigation of CD19 CAR Tcell cytokine release syndrome without attenuation of antileukemic efficacy. Blood. 2019;134(24):2149-58. https://doi.org/10.1182/ blood.2019001463.

49. Sato R, Nasu M. A review of sepsis-induced cardiomyopathy. J Intensive Care. 2015;3(1). https://doi.org/10.1186/s40560-0150112-5.

50. Ahmed T. Cardiovascular risk profile of chimeric antigen receptor T-cell therapy. Cureus. 2020. https://doi.org/10.7759/cureus. 7436.

51. Ganatra S, Carver JR, Hayek SS, Ky B, Leja MJ, Lenihan DJ, et al. Chimeric antigen receptor T-cell therapy for cancer and heart: JACC council perspectives. J Am Coll Cardiol. 2019;74(25): 3153-63. https://doi.org/10.1016/j.jacc.2019.10.049.

52. Lee DW, Kochenderfer JN, Stetler-Stevenson M, Cui YK, Delbrook C, Feldman SA, et al. T cells expressing CD19 chimeric antigen receptors for acute lymphoblastic leukaemia in children and young adults: a phase 1 dose-escalation trial. Lancet.
2015;385(9967):517-28. https://doi.org/10.1016/s0140-6736(14) 61403-3.

53. Schuster SJ, Maziarz RT, Rusch ES, Li J, Signorovitch JE, Romanov VV, et al. Grading and management of cytokine release syndrome in patients treated with tisagenlecleucel in the JULIET trial. Blood Adv. 2020;4(7):1432-9. https://doi.org/10.1182/ bloodadvances.2019001304.

54. Lee DW, Santomasso BD, Locke FL, Ghobadi A, Turtle CJ, Brudno JN, et al. ASTCT consensus grading for cytokine release syndrome and neurologic toxicity associated with immune effector cells. Biol Blood Marrow Transplant. 2019;25(4):625-38. https:// doi.org/10.1016/j.bbmt.2018.12.758.

55. Lee DW, Gardner R, Porter DL, Louis CU, Ahmed N, Jensen M, et al. Current concepts in the diagnosis and management of cytokine release syndrome. Blood. 2014;124(2):188-95. https://doi.org/10. 1182/blood-2014-05-552729.

56. Park JH, Rivière I, Gonen M, Wang X, Sénéchal B, Curran KJ, et al. Long-term follow-up of CD19 CAR therapy in acute lymphoblastic leukemia. N Engl J Med. 2018;378(5):449-59. https://doi. org/10.1056/nejmoa1709919.

57. Porter D, Frey N, Wood PA, Weng Y, Grupp SA. Grading of cytokine release syndrome associated with the CAR T cell therapy tisagenlecleucel. J Hematol Oncol. 2018;11(1). https://doi.org/10. 1186/s13045-018-0571-y.

58. Kotch C, Barrett D, Teachey DT. Tocilizumab for the treatment of chimeric antigen receptor $\mathrm{T}$ cell-induced cytokine release syndrome. Expert Rev Clin Immunol. 2019;15(8):813-22. https://doi. org/10.1080/1744666X.2019.1629904.

Publisher's Note Springer Nature remains neutral with regard to jurisdictional claims in published maps and institutional affiliations. 